\title{
Scenario Based Approach for Load Identification
}

Vigsø, Michael; Tarpø, Marius; Hansen, Jannick Enøe; Brincker, Rune; Georgakis, Christos T.

Published in:

Dynamics of Civil Structures

Link to article, DOI:

10.1007/978-3-319-74421-6_15

Publication date:

2018

Document Version

Peer reviewed version

Link back to DTU Orbit

\section{Citation (APA):}

Vigsø, M., Tarpø, M., Hansen, J. E., Brincker, R., \& Georgakis, C. T. (2018). Scenario Based Approach for Load Identification. In S. Pakzad (Ed.), Dynamics of Civil Structures (Vol. 2, pp. 117-125). Springer. Conference Proceedings of the Society for Experimental Mechanics Series Vol. 2 https://doi.org/10.1007/978-3-319-744216_15

\section{General rights}

Copyright and moral rights for the publications made accessible in the public portal are retained by the authors and/or other copyright owners and it is a condition of accessing publications that users recognise and abide by the legal requirements associated with these rights.

- Users may download and print one copy of any publication from the public portal for the purpose of private study or research.

- You may not further distribute the material or use it for any profit-making activity or commercial gain

- You may freely distribute the URL identifying the publication in the public portal 


\title{
Scenario Based Approach for Load Identification
}

\author{
Michael Vigsø* \\ PhD Student \\ Aarhus University \\ Inge Lehmanns Gade 10 \\ Aarhus, Denmark
}

\author{
Marius Tarpø \\ PhD Student \\ Aarhus University \\ Inge Lehmanns Gade 10 \\ Aarhus, Denmark
}

\author{
Jannick B. Hansen \\ Associate Professor \\ Aarhus University \\ Inge Lehmanns Gade 10 \\ Aarhus, Denmark
}

\author{
Rune Brincker \\ Professor \\ Technical University of Denmark \\ Brovej Building 118 \\ Kgs. Lyngby, Denmark
}

\author{
Christos T. Georgakis \\ Professor \\ Aarhus University \\ Inge Lehmanns Gade 10 \\ Aarhus, Denmark
}

\begin{abstract}
In output only analysis the load identification has been a puzzle for several years. Different techniques have been purposed to cope with the inversion problem that lies within this field. However it has been shown, that most methods struggle to obtain robust and consistent results in cases of modal truncation and noise contaminated signals. In the light of these challenges, a scenario based method is proposed. This approach utilizes model updating along with mode shape expansion to obtain a reliable numerical model of the given structure. Then, by evaluating a series of rational load scenarios, it is possible to obtain a reasonable input identification - both the spatial distribution and the temporal variation of the load. The method is demonstrated numerically and experimentally.
\end{abstract}

Keywords: System Identification, Operational Modal Analysis, Response Estimation, Modal Truncation, FE updating.

\section{NOMENCLATURE}

Vectors are noted by a single underline and matrix by a double underline. Superscript* indicates complex conjugated while superscript ${ }^{\wedge}$ is an approximation.

$\begin{array}{ll}\dagger & \text { Moore Penrose pseudo inverse } \\ \omega_{r} & \text { Natural frequency of mode } r \\ \zeta_{r} & \text { Damping ratio of mode } r \\ \underline{\Phi} & \text { Mode shapes, arranged in columns } \\ \overline{\lambda_{r}} & \text { Pole of mode } r \\ \varepsilon & \text { RMS error } \\ Q_{r} & \text { Modal scaling constant for mode } r \\ f(t) & \text { Force } \\ \underline{f_{0}} & \text { Spatial distribution of load } \\ g(t) & \text { Scaling function of load } \\ \underline{\underline{h}}(t) & \text { Impulse response function }\end{array}$

$y(t) \quad$ Displacements

$\bar{q}(t) \quad$ Modal coordinates

$\underline{Y}(\omega) \quad$ Fourier transformed of $y(t)$

$\underline{C}(\omega) \quad$ Fourier transformed of $\underline{\underline{c}}(t)$

$G(\omega) \quad$ Fourier transformed of $g(t)$

$\underline{\underline{H}}(\omega) \quad$ Frequency response function

${ }^{*}$ Corresponding author 


\section{INTRODUCTION}

Being able to determine the dynamic loading of a mechanical system is of interest in many contexts. In most civil engineering structures, however, it is not feasible to get direct measurements of the dynamic loading and one must rely on indirect methods by measuring the response. Often however, either due to inaccessibility or to limited amount of sensors, the response of the structure is hardly ever available at all desired locations which increases the complexity of the problem [1].

Utilizing the concepts of Operational Modal Analysis, OMA, on a structure, it is possible to extract key parameters of the system, which can be used to reconstruct a model representation [2]. Then by carefully expanding the mathematical formulation of the system, it is possible to estimate the response at locations whereas it is not feasible to get measurements. By using the expanded mode shapes along with the frequencies and damping ratios obtained from the OMA, one can establish a frequency response function of the system. This yields an inverse identification problem where the number of unknowns exceeds the number of equations and a unique solution is not possible to achieve. Methods to cope with this inversion instability have been proposed either through least square schemes [3] or filtering using singular value decomposition [4]. Since complete dynamic characteristics of the system are rarely available, truncation errors and noise can cause the input estimates to be rather non-physical.

This paper presents a way to improve the load estimate by constraining the inversion problem by evaluating a set of pre-defined load scenarios. The hypothesis is that when evaluating the "correct" load scenario this yields a minimum error. The mode shape expansion provides the option to detect loads at locations where the response is not originally recorded.

The method is developed for time invariant systems with linear assumption and small damping.

\section{CONCEPT}

The main idea in this approach is to assume the load can be written as a product between a spatial distribution $f_{0}$ and a time dependent scaling function $g(t) \in \mathbb{R}$.

$$
\underline{f}(t)=\underline{f_{0}} g(t)
$$

There is nothing novel in making this separation of variables as several authors have already published papers including this approach, e.g. [5-10]. However the assumption proves to yield robust solutions for load identification. In the time domain, the response of a multi-degree-of-freedom system can be found as a convolution between the impulse response function and the load.

$$
\underline{y}(t)=\underline{\underline{h}}(t) * \underline{f}(t)
$$

Given that the load $f(t)$ can be written as in equation (1), the response can then be approximated as

$$
\begin{aligned}
\underline{\hat{y}}(t) & =\left(\underline{\underline{h}}(t) \underline{f_{0}}\right) * g(t) \\
& =\underline{c}(t) * g(t)
\end{aligned}
$$

The easiest way to evaluate this convolution is in the frequency domain, so by means of the Fourier transform equation (3) becomes:

$$
\begin{aligned}
\hat{\underline{Y}}(\omega) & =\left(\underline{\underline{H}}(\omega) \underline{f_{0}}\right) G(\omega) \\
& =\underline{C}(\omega) G(\omega)
\end{aligned}
$$

It is then possible to obtain a least square solution for the scaling function $G(\omega)$.

$$
\hat{G}(\omega)=\underline{C}^{\dagger}(\omega) \underline{Y}(\omega)
$$

By making this formulation, one is omitting the challenges associated with inverting the frequency response function $\underline{H}(\omega)$, which very well may be ill posed $[1,4]$.

Then by evaluating eq. (7) and back substituting into eq. (5), it is possible to obtain an estimate of the response of the system given the load distribution, $f_{0}$, which was chosen initially. This can then be compared to the recorded response and $f_{0}$ can be changed until the error has been minimized. 


\section{SIMULATION CASE}

A simulation case is made on a plane cantilever beam. Bernoulli-Euler beam element formulation is used with two degrees of freedom at each node and a distributed mass. Assuming, that the cantilever beam is subjected to loading approaching Gaussian white noise then the modal parameters can be extracted using common OMA approaches [2]. Mode shapes, $\underline{\Phi}$, and associated frequencies, $\omega_{r}$, and damping rations, $\zeta_{r}$, are extracted from the model and are assumed to be of high quality. For the sake of convenience, the damping ratio is assumed to be the same for all modes. Table 1 shows the extracted modal parameters for the first eight modes and their associated damping ratio.

Table 1 Extracted Modal Parameters

\begin{tabular}{lcccccccc}
\hline Mode & 1 & 2 & 3 & 4 & 5 & 6 & 7 & 8 \\
\hline Frequency [Hz] & 8.32 & 52.1 & 146 & 286 & 473 & 707 & 988 & 1320 \\
Damping ratio [\%] & 1.0 & 1.0 & 1.0 & 1.0 & 1.0 & 1.0 & 1.0 & 1.0 \\
\hline
\end{tabular}

In the current simulation case the model is subjected to a load at a single node as shown in Figure 1. Responses are extracted from a limited number of nodes of the model which are considered "measured" or "experimental" responses. Noise is added to the "measured" response in order to stress the algorithm. The level of noise in this case is $100 \mathrm{dBW}$ and no filtering is performed.

Often, the load source will be located at a point where no response is recorded. This is also chosen to be the case in this simulation, where the load is acting exactly between two experimental nodes, see Figure 1.

In order to estimate the loads at locations where data is not available, the measured response must be expanded to the unknown locations. This is done by a transfer to modal coordinates as follows:

$$
\begin{gathered}
\underline{\hat{q}}(t)=\underline{\Phi}^{\dagger} \underline{y}(t) \\
\underline{y_{F E}}(t)=\underline{\underline{\Phi_{F E}}} \underline{\hat{q}}(t),
\end{gathered}
$$

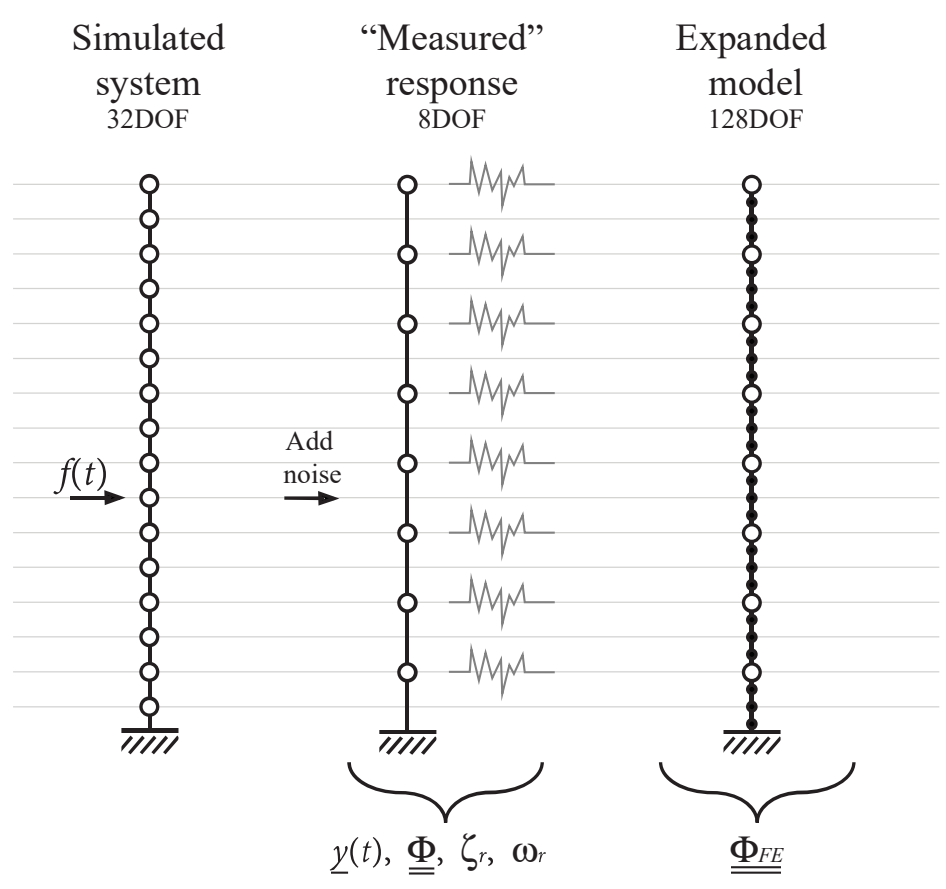

Fig. 1 Simulation case setup. Note: only every second node visible on the expanded model 
where index FE indicates expanded response using Finite Element, FE, mode shapes. Several other expansion techniques are available for this task, e.g. SEREP [11] or LC [12], but in this case adopting the FE mode shapes presuming high correlation between the FE model and the physical model. The size of $\Phi_{F E}$ is determined by the desired resolution during load identification, but is limited to the same number of experimentally determined mode shapes.

A new receptance FRF matrix is formed based on the obtained information from the OMA and an updated FE model.

$$
\underline{\underline{H}}(\omega)=\sum_{r=1}^{N}\left(\frac{Q_{r} \underline{\psi_{r}} \cdot \underline{\psi_{r}^{T}}}{i \omega-\lambda_{r}}+\frac{Q_{r}^{*}{\underline{\psi_{r}}}^{*} \cdot \underline{\psi_{r}^{* T}}}{i \omega-\lambda_{r}^{*}}\right)
$$

where $Q_{r}$ is a modal scaling constant and $\psi_{r}$ is the expanded and mass normalized mode shape $r$.

Letting $\underline{\underline{F_{0}}}$ contain a set of different predefined load distributions $\underline{f_{0}}$. The distributions can be arbitrary chosen but for this case say $\underline{\underline{F_{0}}}$ being equal to the identity matrix meaning that each column corresponds to a single localized force or moment. That is

$$
\underline{\underline{F_{0}}}=\left[\underline{f}^{(1)}, \underline{f}^{(2)} \ldots \underline{f}^{(N)}\right]=\left[\begin{array}{cccc}
1 & 0 & \ldots & 0 \\
0 & 1 & \ldots & 0 \\
\vdots & \vdots & \ddots & \vdots \\
0 & 0 & \ldots & 1
\end{array}\right]_{N \times N}
$$

where $N$ is the total number of free degrees of freedom in the system. Then by evaluating the different distributions, $f_{0}$, one by one it is possible to get an estimate of the approximated response corresponding to that distribution, eq. (5) to (7). Letting the error on the estimate, $\varepsilon$, be quantified as

$$
\varepsilon=\sum_{\text {all DOFs }} R M S(\underline{y}(t)-\underline{\hat{y}}(t))
$$

In Figure 2, the error for each load scenario is shown. The curve for the rotational DOFs is included merely for academic interest as the most likely physical input will be a force rather than a moment. In this case a clear minimum is observed at a force acting at node 28. From Figure 1 it can be seen that node 28 in the expanded model coincide with the position of the load. By selecting the load distribution, $f_{0}$, causing the minimum error and multiplying by the scaling function, $g(t)$, associated with this distribution, it is then possible to get an estimate on the load $f(t)$. The result is shown in Figure 3.

The added noise in the response signal is causing the load estimate to fluctuate around the true value. In the frequency domain this yields a noise floor under which the load cannot be reconstructed.

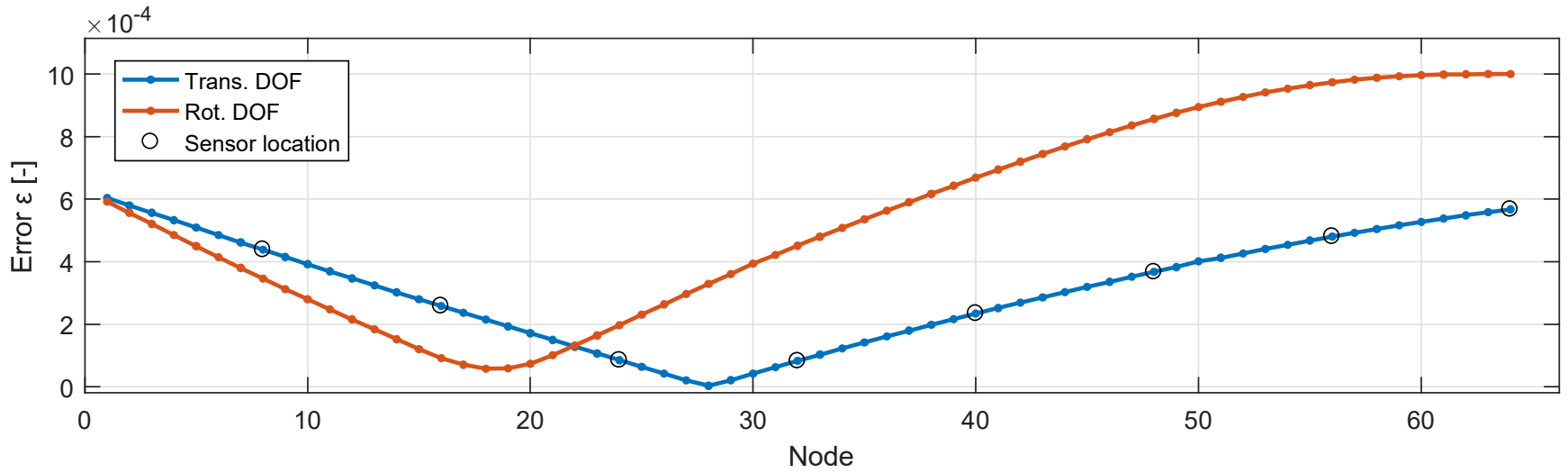

Fig. 2 Calculated error. Each datapoint corresponds to a load scenario in $\underline{F_{0}}$. Every second is loading to a translational DOF and rotational DOF respectively, hence the separation of curves 

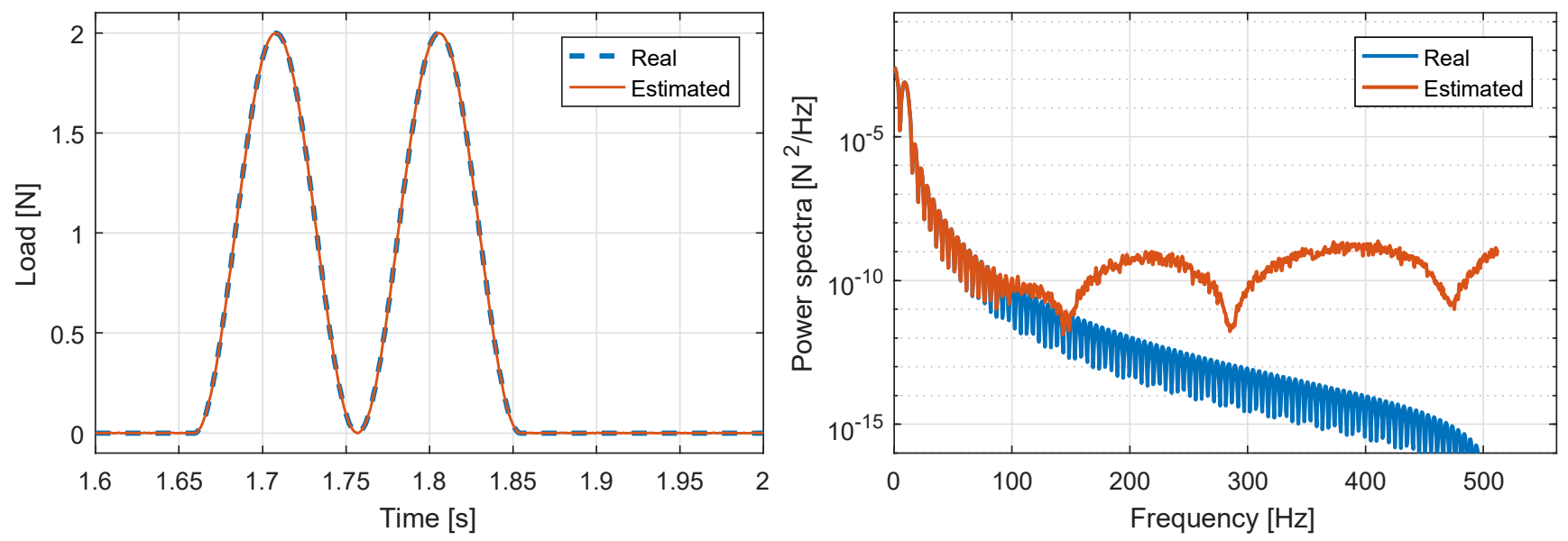

Fig. 3 Time- and frequency domain estimate. 5 modes were included to establish the frequency response function. Power spectra computed using Welch averaging with a block size of $2^{11}$

\section{EXPERIMENTAL CASE}

An experimental case study has been performed at the facility at Aarhus University. A cantilever T-structure made from RHS $40 \times 40 \times 2$ is used for the experiment. The T measures $1.5 \mathrm{~m}$ in height and is $1 \mathrm{~m}$ wide. The $\mathrm{T}$ is clamped to the floor through the base plate seen in Figure 4. Ten uni-axial accelerometers are distributed as shown in Figure 4. Accelerometers used are Brüel \& Kjær type 4508-B 100mV/g.

First, a test with random excitation from compressed air is recorded from which modal parameters are extracted. Identification technique used for this task is the frequency domain decomposition, FDD, [13]. The response is recorded for 300 seconds at a sampling frequency of $1652 \mathrm{~Hz}$. The recordings from the test are represented in terms of singular values from the spectral density matrix, see Figure 5. Note the harmonic peaks at $50 \mathrm{~Hz}$ and $100 \mathrm{~Hz}$ caused by the power grid. Damping ratio is for the first mode estimated as $0.25 \%$ and is assumed to be applicable for all modes. 10 modes were identified in the frequency band of $0-350 \mathrm{~Hz}$ and their frequencies are listed in Table 2.

Secondly an impact hammer is used to generate and record loading of the structure. A Brüel \& Kjær impact hammer type $820622.5 \mathrm{mV} / \mathrm{N}$ is used for the experiment. The hammer is equipped with a soft tip and an impact is made between sensor S03 and S07 in the $y$ direction. The exact position is $12 \mathrm{~cm}$ below the sensor S03 i.e. $1 / 4$ of the distance between the two sensors, see Figure 4 .
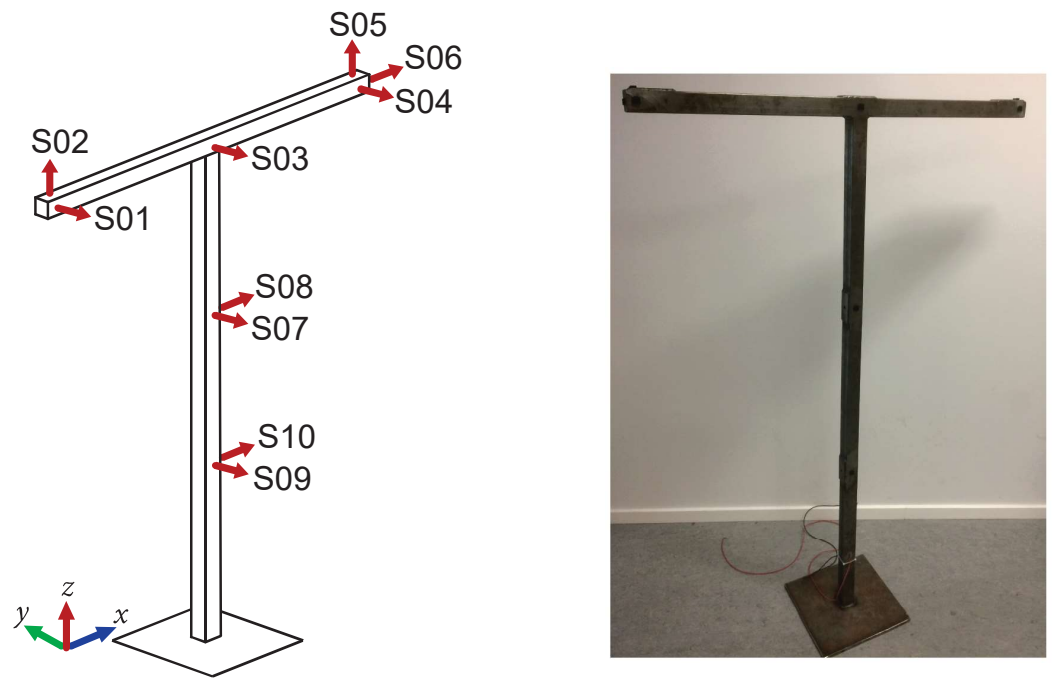

Fig. 4 Left: Schematic sensor layout. Right: Photo of test subject 


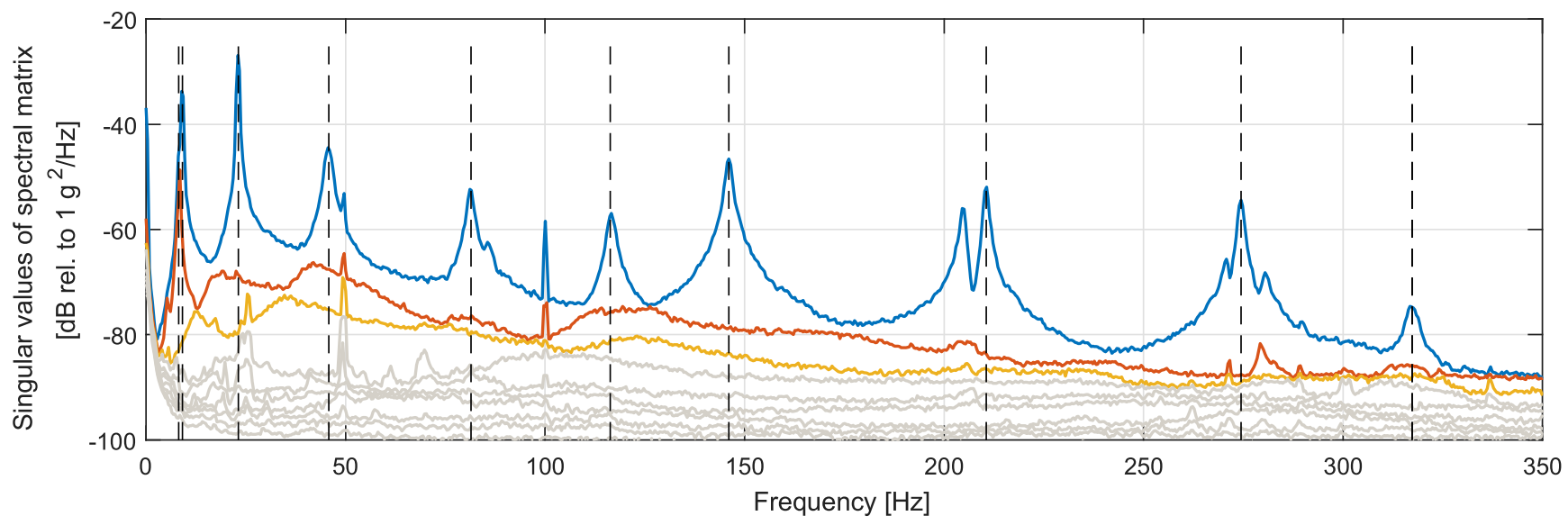

Fig. 5 Singular value decomposition and selected eigenfrequencies. Spectral matrix computed using Welch averaging with a block size of $2^{12}$
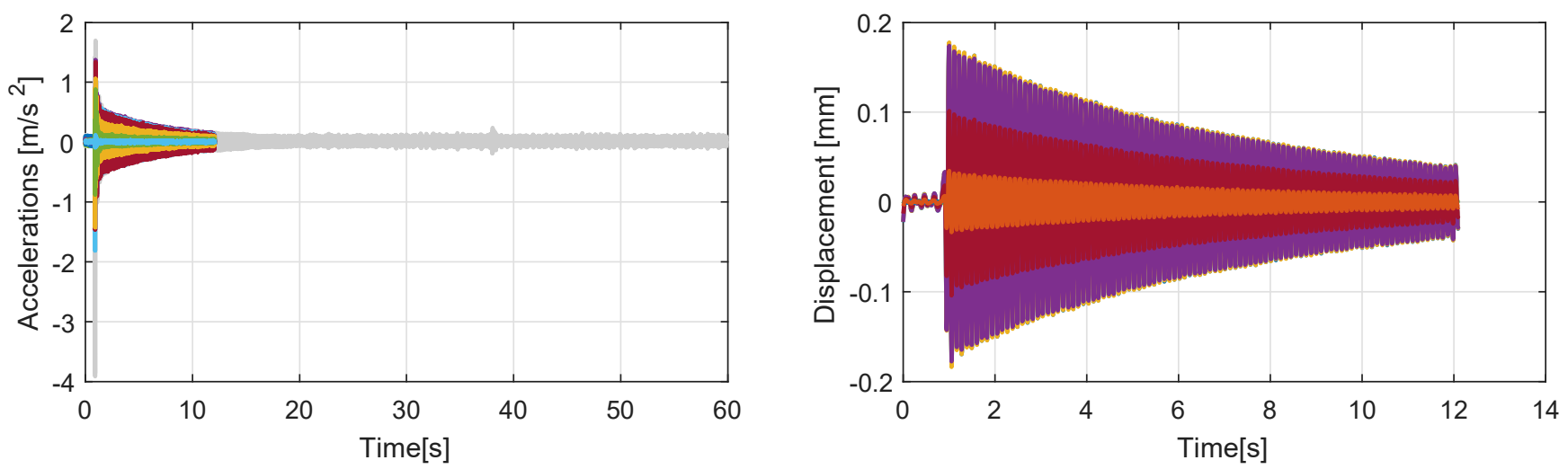

Fig. 6 Impact response shown for all channels

Figure 6 shows the raw acceleration response recorded during the impact. The accelerations are integrated twice in the frequency domain to obtain the displacements. During integration a highpass filter of $5 \mathrm{~Hz}$ is used. In order to improve computational efficiency only $12 / 60 \mathrm{~s}$ are included in the analysis. In addition the signal is decimated with a factor 4 pushing the Nyquist frequency down to $207 \mathrm{~Hz}$ [14].

An FE model is made and updated to increase the correlation of frequencies and mode shapes. The model is made from Bernoulli beam elements with six degrees of freedom at each node and distributed mass. Meshing of the structure is done so that the sensor locations match an FE node. The number of FE nodes are chosen with respect to convergence of the modes of interest and the desired spacial resolution for the successive load identification. The mesh density may be seen in Figure 9.

The model is then updated in two subsequent steps. 1.: Material density increased until the total weight of the FE model corresponds to the physical model. 2.: The rotational stiffness at the base of the T, $R x, R y, R z$ are updated until a best fit for the first 10 modes is achieved. The MAC matrix (after updating) can be seen in Figure 7. The MAC matrix is computed using DOF pairs between the FE model and the experimental DOFs.

Table 2 Natural frequencies

\begin{tabular}{lcccccccccc}
\hline Mode & 1 & 2 & 3 & 4 & 5 & 6 & 7 & 8 & 9 & 10 \\
\hline Frequency OMA [Hz] & 8.17 & 9.08 & 23.1 & 44.8 & 81.4 & 116 & 146 & 211 & 274 & 317 \\
Frequency FE [Hz] & 8.34 & 8.83 & 24.6 & 45.3 & 81.1 & 116 & 147 & 207 & 277 & 315 \\
\hline
\end{tabular}




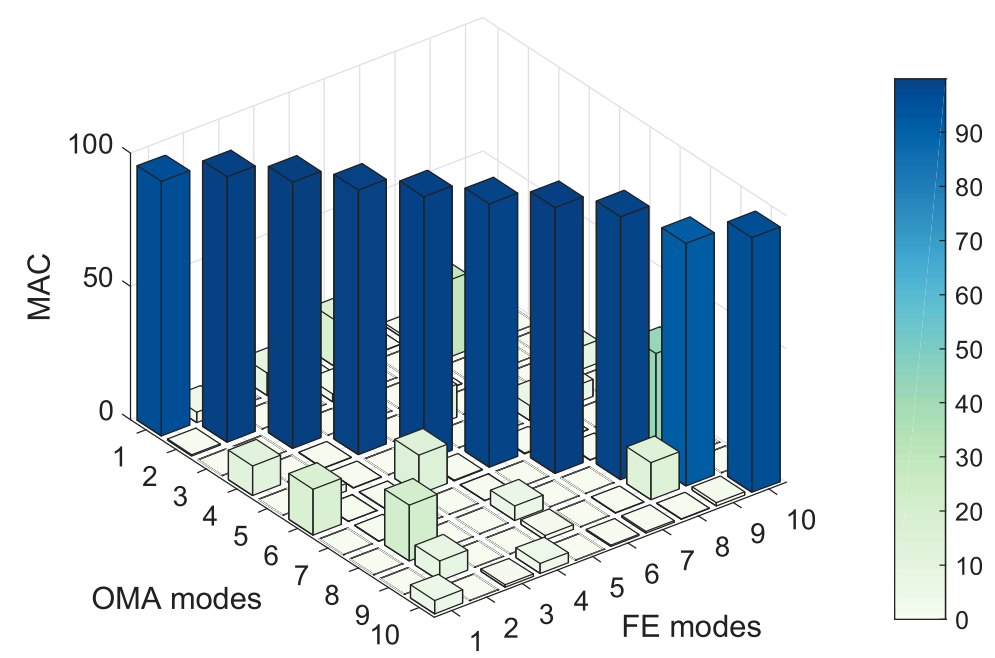

Fig. 7 Modal Assurance after updating

Once an FE model is created and updated to an acceptable level, the frequency response function can yet again be formed using equation (10). The mass normalized mode shapes from the updated FE model are used along with the eigenfrequencies identified from the OMA. All ten mode shapes are included in the process.

When defining the set of possible load distributions, $\underline{F}_{0}$, again the identity matrix is used as a basis. However, this time, omitting the rotational DOFs. Since the expanded model contains 41 nodes, (including the fixed node at the base), this yields in total 120 different load scenarios to be evaluated.

The error associated with each load scenario is shown in Figure 8. The results have been broken into three groups depending on their direction. As seen in Figure 8 impact in the $y$ direction yields much less error than for the other directions. The minimum is more indistinct compared to the simulated case study in Figure 2. However a minimum is observed at the location of impact - node 23.

Since the load distributions used in this case consist of localized forces acting at one node at a time, the errors associated with each distribution can be assimilated with a spacial position and provide a visual feedback on where an impact was most likely to have happen. This is shown in Figure 9.
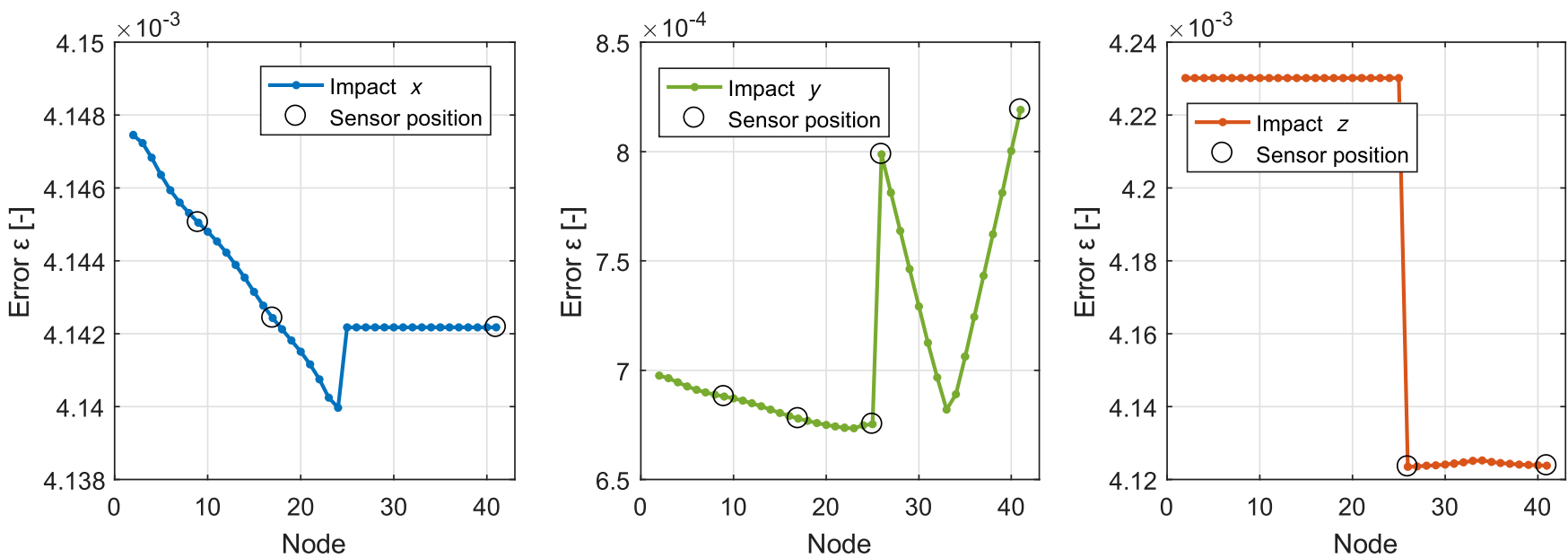

Fig. 8 Calculated error. Node 1 is located at the base of the $\mathrm{T}$ and node 25 is located at the joint 


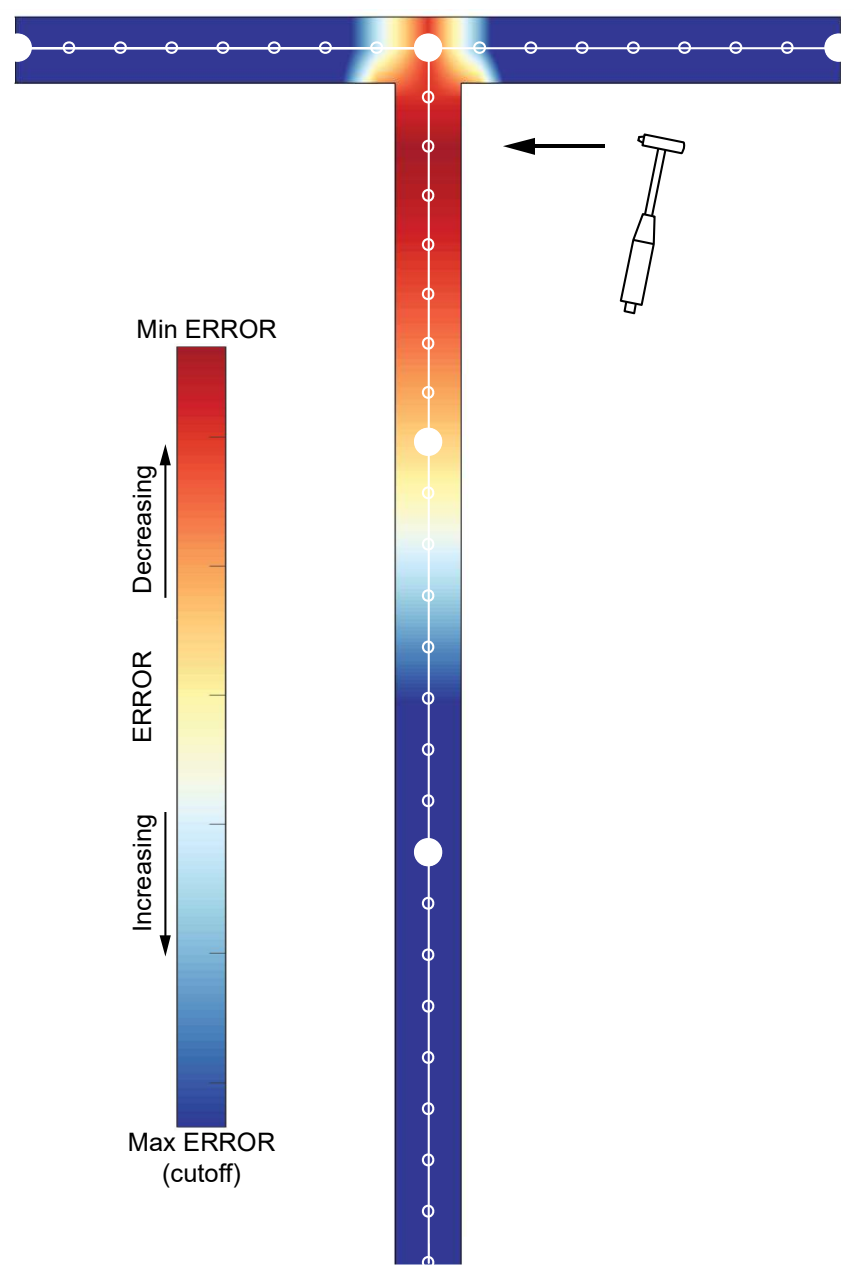

Fig. 9 Re-representation of the calculated error from Figure 8 - $y$ direction impact. Figure indicates at which position the impact was most likely to occur with linear interpolation between nodes. The bold dots indicate sensor position whereas the open dots represent the FE nodes
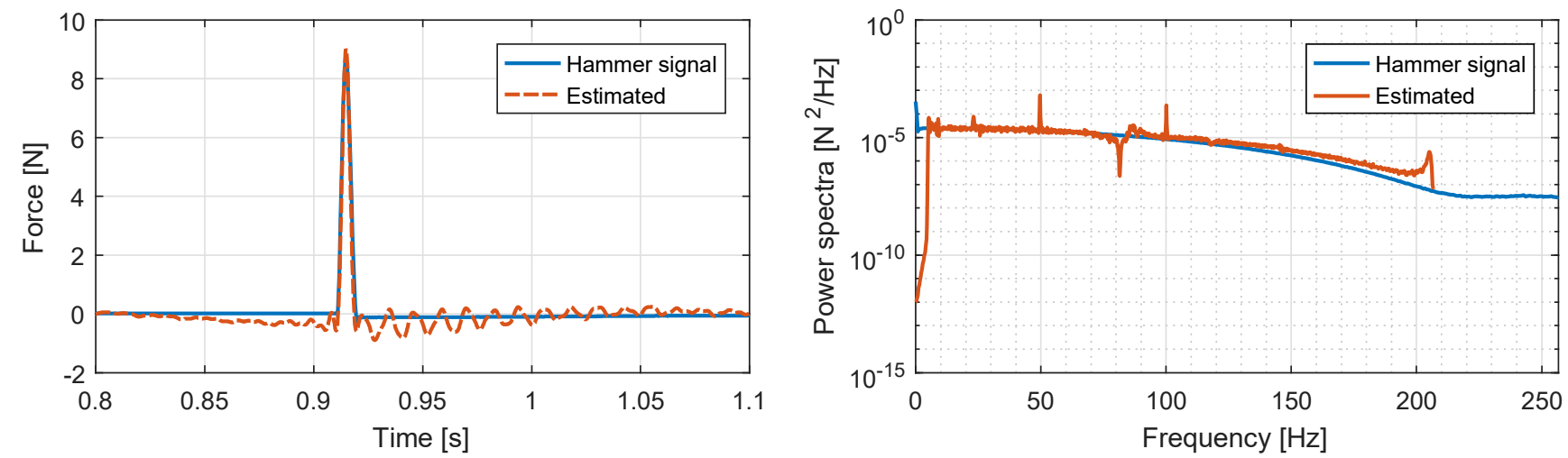

Fig. 10 Identified load. Power spectra computed using Welch averaging with a block size of $2^{11}$

The load distribution causing the minimum error can be multiplied by its scaling function and an estimate for temporal variation can be obtained as it was done in the simulation case study. The result on the load estimate is shown in Figure 10. In the time domain, the estimate follows the trend of the hammer with a peak deviation of 3 percent. It is observed that a negative load is estimated prior to the impact and some ringing afterwards. 
Looking at the response data in Figure 6 it is observed, that the structure is not at complete rest at the time of impact. Since the load estimate is directly related to the response of the structure any ambient vibration will skew the identification of hammer input. In the frequency domain the estimate shows some discrepancy around the resonance frequencies. This may be due to the assumption that all modes bear the same damping ratio.

Noise spikes are observed at 50 and $100 \mathrm{~Hz}$, these are a remaining product of the harmonic noise in the response signal.

\section{CONCLUSION}

A method for indirect load identification has been presented. The method is titled "scenario based" as a keystone to success is having a set of good load distributions to select from. The method was demonstrated by a numerical simulation and a physical experiment. Some of the main observations are recaptured here:

By use of expanded mode shapes, the method shows potential in load identifications even at locations where the response is not recorded immediately. In the two cases the method proves not to yield any false-positive estimates for the position of the impact load, although the estimated location of impact is more outspoken for the numerical study than for the physical experiment. The rank deficiency of the FRF matrix is tamed by assuming a load distribution prior to making a least square fit. This makes the method insensitive to modal truncation, but remains reliant on a well-updated FE model. The noise and harmonic disturbance to the recorded response signal is causing the load identification to deviate but the algorithm remains stable.

Obviously one of the shortcomings to this method is the need for engineering judgement in order to develop a set of reasonable load distributions. Since this method is scenario based it is crucial that a fairly good distribution has been foreseen prior to the analysis otherwise the estimate may be erroneous.

Another challenge to this method arises if the distribution changes with time. Segmenting the response may improve this limitation but further work is needed in this area.

It is fundamental that the response is recorded at the time of loading. I.e. the structure must be at rest in the beginning of a time series otherwise this may alter the load estimates. This assumption seems to be violated to some extent in the physical experiment presented in this paper. From Figure 6 it seems like the T was vibrating from excitation not caused by the hammer.

\section{FUTURE WORK}

In principle there are no limitations to the load distribution and the method could be applied on other cases, where some prior knowledge on loading pattern exists. For instance wave loading on an offshore structure.

Some limiting factors, which have not been covered in great detail, could be a basis for further work. For example, what is the sensitivity of the estimated modal parameters and how will a small change influence the load identification process or how this method will perform on highly damped systems.

\section{ACKNOWLEDGEMENTS}

The authors acknowledge the funding received from Centre for Oil and Gas - DTU/Danish Hydrocarbon Research and Technology Centre (DHRTC).

\section{REFERENCES}

[1] K. Stevens. Force identification problems - an overview. Proceedings of the SEM Spring Conference on Experimental Mechanics, pages 838-844, 1987.

[2] R. Brincker and C. Ventura. Introduction to Operational Modal Analysis. Wiley, 2015.

[3] Y. Liu and W. Shepard. Dynamic force identification based on enhanced least squares and total least-squares scheme in the frequency domain. Journal of Sound and Vibration, Vol: 282:37-60, 2005.

[4] M. Aenlle, R. Brincker, P. Fernández, and A. Fernández. Load estimation from modal parameters. Proceedings of the 2nd International Operational Modal Analysis Conference, Vol. 1:39-50, 2007. 
[5] P. Léger, J. M Ricles, and L. J. Robayo. Reducing modal truncation error in the wave response analysis of offshore structures. Communications in applied numerical methods, Vol: 6:7 - 16, 1990.

[6] K. Maes, A Iliopoulos, W Weijtjens, C Devriendt, and G Lombaert. Dynamic strain estimation for fatigue assessment of and offshore monopile wind turbine using filtering and modal expansion algorithms. Mechanical Systems and Signal Processing, Vol: 76-77:592-611, 2016.

[7] K. Maes, Q.W. Smyth, G. De Roeck, and G. Lombaert. Joint input-state estimation in structural dynamics. Mechanical Systems and Signal Processing, Vol: 70-71:445-466, 2016.

[8] E. Parloo, P. Verboven, P. Guillaume, and M. van Overmeire. Force identification by means of in-operation modal models. Journal of sound and vibration, Vol. 262:161-173, 2002.

[9] B. Wang and C. Chiu. Determination of unknown impact force acting on a simply supported beam. Mechanical Systems and Signal Processing, Vol: 17 No 3:683-704, 2003.

[10] E. Lourens, E. Reynders, G. De Roeck, G Degrande, and G Lombaert. An argumented Kalman filter for force identification in structural dynamics. Mechanical Systems and Signal Processing, Vol: 27:446-460, 2012.

[11] J. O'Callahan, P. Avitabile, and R. Riemer. System equivalent reduction expansion process. Proceedings of the 7th International Modal Analysis Conference (IMAC), 1989.

[12] R. Brincker, A. Skafte, M. Anelle, A. Sestieri, W. D'Ambrogio, and A. Canteli. A local correspondence principle for mode shapes in structural dynamics. Mechanical Systems and Signal Processing, Vol: 45:91-104, 2014.

[13] R. Brincker, L. Zhang, and P. Andersen. Modal identification from ambient responses using frequency domain decomposition. Proceedings of the International Modal Analysis Conference, IMAC, pages 625-630, 2000.

[14] R. Lyons. Understanding Digital Signal Processing. Pearson Education, 2011. 